QUARTERLY OF APPLIED MATHEMATICS

VOLUME LXX, NUMBER 2

JUNE 2012, PAGES 345-356

S 0033-569X(2012)01254-5

Article electronically published on February 29, 2012

\title{
EXISTENCE OF SOLUTIONS TO THE RIEMANN PROBLEM FOR A CLASS OF HYPERBOLIC CONSERVATION LAWS EXHIBITING A PARABOLIC DEGENERACY
}

\author{
BY \\ HIROKI OHWA \\ Graduate School of Education, Waseda University, 1-6-1 Nishi-Waseda, Shinjuku-ku, Tokyo, \\ 169-8050, Japan
}

\begin{abstract}
This paper contains a proof of the existence of solutions to the Riemann problem for a class of $2 \times 2$ hyperbolic conservation laws exhibiting a parabolic degeneracy. The method used in this paper is based upon the vanishing viscosity approach. This approach enables us to establish the existence of solutions to the Riemann problem for those systems which do not satisfy the genuine nonlinearity condition and the shock admissibility condition.
\end{abstract}

1. Introduction. We consider a class of $2 \times 2$ hyperbolic conservation laws with parabolic degeneracy defined by the equations

$$
u_{t}+(f(u)-v)_{x}=0, \quad v_{t}+g(u)_{x}=0, \quad t>0,-\infty<x<\infty,
$$

where $u$ and $v$ are functions of $t$ and $x$, and $f$ and $g$ are smooth functions of $u$. The Riemann problem for system (1.1) consists in finding a solution of (1.1) with piecewise constant initial data of the form

$$
(u(0, x), v(0, x))= \begin{cases}\left(u_{-}, v_{-}\right), & x<0 \\ \left(u_{+}, v_{+}\right), & x>0\end{cases}
$$

A system

$$
U_{t}+F(U)_{x}=0
$$

with $U=(u, v), F(U)=\left(f_{1}(U), f_{2}(U)\right)$, is said to possess a parabolic degeneracy on a curve $\Sigma$ in the $U$-plane if the eigenvalues $\lambda_{1}(U)$ and $\lambda_{2}(U)$ of the matrix $A=\operatorname{grad} F$ are

Received October 4, 2010.

2010 Mathematics Subject Classification. Primary 35L45, 35L65.

Key words and phrases. Conservation laws, nonlinear wave equation, the Riemann problem, the vanishing viscosity approach.

E-mail address: ohwa-hiroki@suou.waseda.jp

(C) 2012 Brown University Reverts to public domain 28 years from publication 
real for all $U$ in $\mathbb{R}^{2}$, distinct for $U$ off $\Sigma$, and coincident with a single common eigenvector when $U$ lies on $\Sigma$. The prototype of system (1.3) with a parabolic degeneracy is

$$
u_{t}=v_{x}, \quad v_{t}=\left(\frac{u^{3}}{3}\right)_{x} .
$$

This system arises from the nonlinear wave equation $u_{t t}=\left(c^{2} u_{x}\right)_{x}$ with $c=u$. The parabolic degeneracy occurs at $u=0$ and corresponds physically to zero sound speed. Moreover, a modified wave equation of the form

$$
u_{t}+\left(\gamma u^{2}-v\right)_{x}=0, \quad v_{t}-\left(\frac{u^{3}}{3}\right)_{x}=0,
$$

with $\gamma>0$, has a parabolic degeneracy. We remark that system (1.1) includes systems (1.4) and (1.5) .

The classical method of solution to the Riemann problem requires the fact that the Hugoniot curves always consist of only shock curves which, for every point $U_{0} \in \mathbb{R}^{2}$, are simple arcs extending from $U_{0}$ to infinity and satisfying the Lax entropy condition (see [13 for the Lax entropy condition). The method is called the shock curve approach (cf. [17] and [18]). By using the shock curve approach, a lot of papers have been written on the existence and uniqueness of entropy solutions (in the sense of Lax, Liu, et al.) to the Riemann problem (e.g. [10], 11], [12, 14], 15], 16, 17], 18], 20, and others). However, since the fact can be established only if the shock admissibility conditions are known a priori, it is not easy to use the shock curve approach. For this reason, in general, the approach has been applied so far only to genuinely nonlinear systems with additional conditions.

It must be noted that if $d g(u) / d u \leq 0$, then the matrix $A=\operatorname{grad} F$ with $F=(f(u)-$ $v, g(u))$ has the real eigenvalues $\lambda_{1}(U) \leq \lambda_{2}(U)$. By using the shock curve approach, Keyfitz and Kranzer [12] prove the existence and uniqueness of entropy solutions (in the sense of Lax) to the Riemann problem for system (1.1) satisfying

$$
\begin{gathered}
g(0)=\left.\frac{d g(u)}{d u}\right|_{u=0}=\left.\frac{d^{2} g(u)}{d u^{2}}\right|_{u=0}=0,\left.\quad \frac{d^{3} g(u)}{d u^{3}}\right|_{u=0}<0, \\
\frac{d g(u)}{d u}<0 \quad \text { for all } u \neq 0, \\
\operatorname{sgn} \frac{d^{2} g(u)}{d u^{2}}=-\operatorname{sgn} u \quad \text { for all } u \neq 0, \\
f(0)=\left.\frac{d f(u)}{d u}\right|_{u=0}=0, \quad \frac{d^{2} f(u)}{d u^{2}} \geq 0, \\
\operatorname{sgn}\left(\frac{d^{2} f(u)}{d u^{2}} \lambda_{i}-\frac{d^{2} g(u)}{d u^{2}}\right)=\operatorname{sgn} u, \\
\frac{f(u)}{g(u)} \rightarrow 0 \quad \text { as }|u| \rightarrow 0, \\
\left|\frac{d g(u)}{d u}\right| \rightarrow \infty \quad \text { as }|u| \rightarrow \infty,
\end{gathered}
$$

and the shock admissibility condition (see conditions $(2.22 \mathrm{a}),(2.22 \mathrm{~b})$ in [12] for the shock admissibility condition). Note that condition (1.10) implies the genuine nonlinearity condition. It is clear that system (1.4) satisfies these conditions. However, system (1.5) 
does not always satisfy these conditions, since the shock admissibility condition is not satisfied for sufficiently large $\gamma$.

The purpose of this paper is to give a proof of the existence of solutions to the Riemann problem for system (1.1) satisfying the following conditions:

$$
\begin{gathered}
\left.\frac{d g(u)}{d u}\right|_{u=0}=\left.\frac{d^{2} g(u)}{d u^{2}}\right|_{u=0}=0,\left.\quad \frac{d^{3} g(u)}{d u^{3}}\right|_{u=0}<0, \\
\frac{d g(u)}{d u}<0 \quad \text { for all } u \neq 0, \\
|g(u)| \rightarrow \infty \quad \text { as }|u| \rightarrow \infty .
\end{gathered}
$$

It should be noticed that we do not require the genuine nonlinearity condition and the shock admissibility condition. Consequently, this statement gives us the fact that the Riemann problem for system (1.5) with all $\gamma>0$ possesses solutions. The method used in this paper is based upon the vanishing viscosity approach introduced in [2], 3], 4, [5] and [21. See [6], [7, [8, 9] and [19] for a study of the vanishing viscosity approach to a mixed hyperbolic-elliptic system. This approach enables us to establish the existence of solutions to the Riemann problem for system (1.1) satisfying (1.13), (1.14) and (1.15). It is well known (cf. [2]) that if system (1.1) endowed an entropy $\eta(u, v)$ with entropy flux $q(u, v)$, then the solutions which are generated by this approach satisfy the following entropy inequality in the distributional sense (see [1] for the entropy inequality):

$$
\eta(u, v)_{t}+q(u, v)_{x} \leq 0 .
$$

The idea of the vanishing viscosity approach is to construct the solution to the Riemann problem as the $\epsilon \rightarrow 0+$ limit of the solutions of

$$
u_{t}+(f(u)-v)_{x}=\epsilon t u_{x x}, \quad v_{t}+g(u)_{x}=\epsilon t v_{x x}, \quad t>0,-\infty<x<\infty
$$

with initial condition (1.2). The unconventional form of the viscosity operator on the right-hand side of (1.17) has been adopted so that the invariance property of (1.1) under the transformation $(t, x) \mapsto(a t, a x), a>0$, is preserved by (1.17). As a consequence of this invariance property, the solution of (1.17), (1.2) is a function $\left(u_{\epsilon}(x / t), v_{\epsilon}(x / t)\right)$ of the single variable $\xi=x / t$, where $\left(u_{\epsilon}(x / t), v_{\epsilon}(x / t)\right)$ is the solution to the boundary-value problem

$$
\begin{array}{r}
\epsilon u_{\epsilon}^{\prime \prime}(\xi)=\left(f\left(u_{\epsilon}(\xi)\right)-v_{\epsilon}(\xi)\right)^{\prime}-\xi u_{\epsilon}(\xi), \quad \epsilon v_{\epsilon}^{\prime \prime}(\xi)=g\left(u_{\epsilon}(\xi)\right)^{\prime}-\xi v_{\epsilon}(\xi), \\
\left(u_{\epsilon}(-\infty), v_{\epsilon}(-\infty)\right)=\left(u_{-}, v_{-}\right), \quad\left(u_{\epsilon}(\infty), v_{\epsilon}(\infty)\right)=\left(u_{+}, v_{+}\right),
\end{array}
$$

where ' denotes differentiation with respect to $\xi$. Therefore, this approach is first to show that for every fixed $\epsilon>0$, the boundary-value problem (1.18), (1.19) has a solution $\left(u_{\epsilon}(\xi), v_{\epsilon}(\xi)\right)$ and then to prove that, as $\epsilon \rightarrow 0+,\left(u_{\epsilon}(\xi), v_{\epsilon}(\xi)\right)$ converges to $(u(\xi), v(\xi))$, where $(u(\xi), v(\xi))$ is the solution to the Riemann problem for system (1.1) satisfying (1.13), (1.14) and (1.15).

We now mention the construction in this paper. Section 2 is devoted to proving the existence of solutions to (1.18), (1.19) under the assumptions of (1.13) and (1.14). It should be noticed that we do not require condition (1.15) in the proof of the existence theorem. In Section 3 we prove that the solutions to (1.18), (1.19) generate solutions of the Riemann problem for system (1.1) satisfying (1.13), (1.14) and (1.15). 
2. Existence of solutions to (1.18), (1.19). In this section, we prove the following result on the existence of solutions to (1.18), (1.19) under the assumptions of (1.13) and (1.14):

Theorem 2.1. Assume that conditions (1.13) and (1.14) are satisfied. Then, for every $\epsilon>0$, there exists a solution to (1.18), (1.19).

This theorem follows from the following theorem, which is shown in Theorem 3.1 of Dafermos 2, under the assumption that we have a priori bounds on the solution:

Theorem 2.2. Consider the two-parameter family of the boundary-value problems

$$
\begin{gathered}
\epsilon u^{\prime \prime}(\xi)=\mu(f(u(\xi))-v(\xi))^{\prime}-\xi u(\xi), \quad \epsilon v^{\prime \prime}(\xi)=\mu g(u(\xi))^{\prime}-\xi v(\xi), \\
(u(-L), v(-L))=\left(\mu u_{-}, \mu v_{-}\right), \quad(u(L), v(L))=\left(\mu u_{+}, \mu v_{+}\right),
\end{gathered}
$$

with parameters $L \geq 1, \mu \in[0,1]$. Assume that there exists a positive constant $M$ depending at most on $\epsilon, f, g,\left(u_{-}, v_{-}\right),\left(u_{+}, v_{+}\right)$(and thus independent of $L$ and $\mu$ ) such that any solution $(u(\xi), v(\xi))$ to (2.1), (2.2) satisfies

$$
\sup _{(-L, L)}|u(\xi)| \leq M, \sup _{(-L, L)}|v(\xi)| \leq M .
$$

Then, for every $\epsilon>0$, there exists a solution to (1.18), (1.19).

From Theorem 2.2 it is sufficient for the proof of Theorem 2.1 to prove the a priori estimate (2.3). In proving estimate (2.3), one is helped by the following theorem, which asserts that one of the components of any solution to (2.1), (2.2) is monotone and the other component is monotone or bell-shaped:

Theorem 2.3. Assume that conditions (1.13) and (1.14) are satisfied and let $(u(\xi), v(\xi))$ be a solution to (2.1), (2.2) with $1 \leq L \leq \infty$. Then, one of the following holds:

(a) Both $u(\xi)$ and $v(\xi)$ are constant on $(-L, L)$.

(b) $u(\xi)$ is a strictly increasing (or decreasing) function with no critical point in $(-L, L)$, while $v(\xi)$ has, at most, one critical point, at which $v(\xi)$ necessarily must attain a maximum (or minimum), in $(-L, L)$.

(c) $u(\xi)$ has, at most, one critical point, at which $u(\xi)$ necessarily must attain a maximum (or minimum), in $(-L, L)$, while $v(\xi)$ is a strictly increasing (or decreasing) function with no critical point in $(-L, L)$.

Proof. Note that when $\mu=0$, a simple computation shows that both $u(\xi)$ and $v(\xi)$ are constant on $(-L, L)$. Suppose now that $\mu>0$ and let $(u(\xi), v(\xi))$ be a nonconstant solution to (2.1), (2.2) on $(-L, L)$.

First, we investigate the properties of $u(\xi)$. Suppose that $\tau$ is a critical point of $u(\xi)$. We then have

$$
\epsilon u^{\prime \prime}(\tau)=\mu\left(\left.\frac{d f(u)}{d u}\right|_{\xi=\tau} u^{\prime}(\tau)-v^{\prime}(\tau)\right)-\tau u^{\prime}(\tau)=-\mu v^{\prime}(\tau)
$$

so that there are the following three possibilities of behaviors at $\xi=\tau$ : (I) $u^{\prime \prime}(\tau)>0$, $v^{\prime}(\tau)<0$; (II) $u^{\prime \prime}(\tau)<0, v^{\prime}(\tau)>0$; (III) $u^{\prime \prime}(\tau)=0, v^{\prime}(\tau)=0$. However, by the uniqueness of the solution to (2.1), (2.2) (cf. Lemma 4.1 in [2]), case (III) corresponds to 
$(u(\xi), v(\xi))$ being constant on $(-L, L)$, which implies a contradiction. Therefore, if $\tau$ is a critical point of $u(\xi)$, then we have either (I) or (II). In other words, for the nonconstant function $u(\xi), u(\xi)$ must attain either a maximum or a minimum at the critical point $\tau$.

Next, we investigate the properties of $v(\xi)$. Suppose that $\tau$ is a critical point of $v(\xi)$. If

$$
\left.\frac{d g(u)}{d u}\right|_{\xi=\tau}<0,
$$

then it follows from arguments similar to the case of $u(\xi)$ that there are the following two possibilities of behaviors at $\xi=\tau:\left(\mathrm{I}^{\prime}\right) v^{\prime \prime}(\tau)>0, u^{\prime}(\tau)<0 ;(\mathrm{II}) v^{\prime \prime}(\tau)<0, u^{\prime}(\tau)>0$, while if

$$
\left.\frac{d g(u)}{d u}\right|_{\xi=\tau}=0
$$

then, by conditions (1.13) and (1.14), we have

$$
\epsilon v^{(4)}(\tau)=\left.\mu \frac{d^{3} g(u)}{d u^{3}}\right|_{\xi=\tau}\left(u^{\prime}(\tau)\right)^{3}
$$

so that there are the following two possibilities of behaviors at $\xi=\tau$ : $\left(\mathrm{I}^{\prime \prime}\right) v^{(4)}(\tau)>0$, $u^{\prime}(\tau)<0 ;\left(\mathrm{II}^{\prime \prime}\right) v^{(4)}(\tau)<0, u^{\prime}(\tau)>0$. Therefore, for the nonconstant function $v(\xi)$, $v(\xi)$ must attain either a maximum or a minimum at the critical point $\tau$.

We now prove that the nonconstant function $u(\xi)$ has at most one critical point in $(-L, L)$ by contradiction. Assume that there exist two different critical points, denoted by $\xi_{1}, \xi_{2}$ with $-L<\xi_{1}<\xi_{2}<L$, of $u(\xi)$. Then it follows from (I) and (II) that $u(\xi)$ attains a maximum (or minimum) at $\xi_{1}$ and attains a minimum (or maximum) at $\xi_{2}$, and $u^{\prime}(\xi)<0$ (or $u^{\prime}(\xi)>0$ ) for $\left(\xi_{1}, \xi_{2}\right)$. As shown above, we have $v^{\prime}\left(\xi_{1}\right)>0$ (or $\left.v^{\prime}\left(\xi_{1}\right)<0\right)$ and $v^{\prime}\left(\xi_{2}\right)<0$ (or $v^{\prime}\left(\xi_{2}\right)>0$ ) and this implies that $v(\xi)$ attains a maximum (or minimum) at some point $\tilde{\tau} \in\left(\xi_{1}, \xi_{2}\right)$. But, then we have $u^{\prime}(\tilde{\tau})>0$ (or $\left.u^{\prime}(\tilde{\tau})<0\right)$, which is a contradiction. By arguments similar to the case of $u(\xi)$, we can prove that the nonconstant function $v(\xi)$ has at most one critical point in $(-L, L)$.

Finally, we prove that among the nonconstant functions $u(\xi)$ and $v(\xi)$, at most one of them has critical points in $(-L, L)$ by contradiction. Assume that $\xi_{1}$ is a critical point of $u(\xi)$ and $\xi_{2}$ is a critical point of $v(\xi)$. The above arguments show that $u(\xi)$ and $v(\xi)$ cannot attain extrema at the same point. Without loss of generality, we can assume that $\xi_{1}<\xi_{2}$. By the above discussion, if $u(\xi)$ attains a maximum (or minimum) at $\xi_{1}$, then we have $v^{\prime}\left(\xi_{1}\right)>0$ (or $v^{\prime}\left(\xi_{1}\right)<0$ ) so that $v(\xi)$ must attain a maximum (or minimum) at $\xi_{2}$. However, in this case, we also have $u^{\prime}\left(\xi_{2}\right)>0$ (or $u^{\prime}\left(\xi_{2}\right)<0$ ), which means that $u(\xi)$ must attain a minimum (or maximum) at $\xi_{1}$. This implies a contradiction. Thus the proof of Theorem 2.3 is complete.

By Theorem 2.3, at least one of $u(\xi), v(\xi)$ is monotone. If both $u(\xi)$ and $v(\xi)$ are monotone, then the a priori estimate (2.3) clearly holds. Therefore, it is sufficient for the proof of estimate (2.3) to deal with the following two cases:

CASE 1. $u(\xi)$ is strictly increasing (or decreasing) on $(-L, L)$, while $v(\xi)$ is strictly increasing (or decreasing) on $(-L, \tau)$, attains a maximum (or minimum) at $\tau$, and is strictly decreasing (or increasing) on $(\tau, L)$. 
CASE 2. $u(\xi)$ is strictly increasing (or decreasing) on $(-L, \tau)$, attains a maximum (or minimum) at $\tau$, and is strictly decreasing (or increasing) on $(\tau, L)$, while $v(\xi)$ is strictly increasing (or decreasing) on $(-L, L)$.

The following proposition plays an important role in proving various estimates on the solution:

Proposition 2.4. Assume that conditions (1.13) and (1.14) are satisfied and let $(u(\xi), v(\xi))$ be a solution to (2.1), (2.2) with $1 \leq L \leq \infty$. Moreover, denote by $\bar{u}=\max \left\{\left|u_{-}\right|,\left|u_{+}\right|\right\}$and $\bar{v}=\max \left\{\left|v_{-}\right|,\left|v_{+}\right|\right\}$the maximums of $\left|u_{ \pm}\right|$and $\left|v_{ \pm}\right|$respectively. Then, we have the following:

(i) If $u(\xi)$ is strictly increasing on $(-L, L)$, while $v(\xi)$ is strictly increasing on $(-L, \tau)$, attains a maximum at $\tau$, and is strictly decreasing on $(\tau, L)$, then the following inequalities hold for some constant $N \geq 0$ which depends solely on $g, u_{-}, u_{+}$:

$$
\begin{gathered}
-\bar{u} \leq u(\xi) \leq \bar{u} \quad \text { for all } \xi \in[-L, L], \\
-\bar{v} \leq v(\xi) \leq \bar{v}+\frac{N}{|\xi-\tau|} \quad \text { for all } \xi \in[-L, L] \backslash \tau .
\end{gathered}
$$

(ii) If $u(\xi)$ is strictly decreasing on $(-L, L)$, while $v(\xi)$ is strictly decreasing on $(-L, \tau)$, attains a minimum at $\tau$, and is strictly increasing on $(\tau, L)$, then the following inequalities hold for some constant $N \geq 0$ which depends solely on $g, u_{-}, u_{+}$:

$$
\begin{gathered}
-\bar{u} \leq u(\xi) \leq \bar{u} \quad \text { for all } \xi \in[-L, L], \\
-\bar{v}-\frac{N}{|\xi-\tau|} \leq v(\xi) \leq \bar{v} \quad \text { for all } \xi \in[-L, L] \backslash \tau .
\end{gathered}
$$

(iii) If $u(\xi)$ is strictly increasing on $(-L, \tau)$, attains a maximum at $\tau$, and is strictly decreasing on $(\tau, L)$, while $v(\xi)$ is strictly increasing on $(-L, L)$, then the following inequalities hold:

$$
\begin{array}{cc}
-\bar{u} \leq u(\xi) \leq \bar{u}+\frac{2 \bar{v}}{|\xi-\tau|} & \text { for all } \xi \in[-L, L] \backslash \tau \\
-\bar{v} \leq v(\xi) \leq \bar{v} & \text { for all } \xi \in[-L, L]
\end{array}
$$

(iv) If $u(\xi)$ is strictly decreasing on $(-L, \tau)$, attains a minimum at $\tau$, and is strictly increasing on $(\tau, L)$, while $v(\xi)$ is strictly decreasing on $(-L, L)$, then the following inequalities hold:

$$
\begin{aligned}
& -\bar{u}-\frac{2 \bar{v}}{|\xi-\tau|} \leq u(\xi) \leq \bar{u} \quad \text { for all } \xi \in[-L, L] \backslash \tau, \\
& -\bar{v} \leq v(\xi) \leq \bar{v} \quad \text { for all } \xi \in[-L, L] .
\end{aligned}
$$

Proof. We only prove (i), because (ii), (iii) and (iv) are proved by arguments similar to the proof of $(\mathrm{i})$.

Since inequality (2.4) clearly holds, we prove inequality (2.5). In order to prove inequality (2.5), it is necessary to prove the following inequality:

$$
\int_{\alpha}^{\beta} v(\xi) d \xi \leq(\beta-\alpha) \bar{v}+N \quad \text { for every interval }(\alpha, \beta) \subset(-L, L) \text {. }
$$


We now prove inequality (2.12). If $v(\alpha)>\bar{v}$, then we set

$$
\eta=\sup \{\xi \in[-L, \alpha): v(\xi) \leq \bar{v}\} .
$$

Note that this set is nonempty in view of the definition of $\bar{v}$. On the other hand, if $v(\alpha) \leq \bar{v}$, then we set

$$
\eta=\inf \{\xi \in(\alpha, \beta): v(\xi) \geq \bar{v}\} .
$$

Note also that if this set is empty, then inequality (2.12) is satisfied for any $N \geq 0$. Similarly, if $v(\beta)>\bar{v}$, then we set

$$
\theta=\inf \{\xi \in(\beta, L]: v(\xi) \leq \bar{v}\}
$$

while if $v(\beta) \leq \bar{v}$, then we set

$$
\theta=\sup \{\xi \in(\alpha, \beta): v(\xi) \geq \bar{v}\} .
$$

By the choices of $\eta$ and $\theta$, we have $v^{\prime}(\eta) \geq 0, v^{\prime}(\theta) \leq 0$ and

$$
\int_{\alpha}^{\beta}[v(\xi)-\bar{v}] d \xi \leq \int_{\eta}^{\theta}[v(\xi)-\bar{v}] d \xi=-\int_{\eta}^{\theta} \xi v^{\prime}(\xi) d \xi .
$$

Therefore, integrating the second equation in (2.1) over $(\eta, \theta)$, and using $v^{\prime}(\eta) \geq 0$, $v^{\prime}(\theta) \leq 0$ and (2.13), we obtain

$$
\int_{\alpha}^{\beta} v(\xi) d \xi \leq(\beta-\alpha) \bar{v}+\mu g(u(\eta))-\mu g(u(\theta)) \leq(\beta-\alpha) \bar{v}+N .
$$

Therefore, inequality (2.12) is proved.

From inequality (2.12) we can easily check inequality (2.5). Indeed, if $\xi<\tau$, then we have

$$
v(\xi)=\frac{1}{\tau-\xi} \int_{\xi}^{\tau} v(\xi) d \zeta \leq \frac{1}{\tau-\xi} \int_{\xi}^{\tau} v(\zeta) d \zeta \leq \bar{v}+\frac{N}{\tau-\xi},
$$

while if $\xi>\tau$, then we have

$$
v(\xi)=\frac{1}{\xi-\tau} \int_{\tau}^{\xi} v(\xi) d \zeta \leq \frac{1}{\xi-\tau} \int_{\tau}^{\xi} v(\zeta) d \zeta \leq \bar{v}+\frac{N}{\xi-\tau} .
$$

Thus the proof of inequality (2.5) is complete.

We now prove Theorem 2.1 by proving estimates (2.3) in Cases 1 and 2 .

First, we prove estimate (2.3) in Case 1 . We only prove the case that $u(\xi)$ is strictly increasing on $(-L, L)$, because the case that $u(\xi)$ is strictly decreasing on $(-L, L)$ is proved by arguments similar to the proof of the case that $u(\xi)$ is strictly increasing on $(-L, L)$. Note that when $u(\xi)$ is strictly increasing on $(-L, L), v(\xi)$ is strictly increasing on $(-L, \tau)$, attains a maximum at $\tau$, and is strictly decreasing on $(\tau, L)$. Thus, in this case, it is sufficient to estimate $v(\tau)$ from above. In estimating $v(\tau)$ from above, we assume that $\tau \geq 0$, because the case of $\tau<0$ is proved by arguments similar to the case of $\tau \geq 0$. 
Integrating the second equation of (2.1) over $(\tau, L)$ and using $v^{\prime}(\tau)=0$ and $v^{\prime}(L) \leq 0$, we obtain

$$
-\int_{\tau}^{L} \xi v^{\prime}(\xi) d \xi \leq \mu g(u(\tau))-\mu g\left(\mu u_{+}\right) .
$$

Hence, for any $\zeta \geq \max \{1, \tau\}$, we have

$$
\begin{aligned}
v(\zeta) & =\mu v_{+}-\int_{\zeta}^{L} v^{\prime}(\xi) d \xi \\
& \leq \mu v_{+}-\int_{\tau}^{L} \xi v^{\prime}(\xi) d \xi \\
& \leq \mu v_{+}+\mu g(u(\tau))-\mu g\left(\mu u_{+}\right) .
\end{aligned}
$$

Noting that $\bar{u}=\max \left\{\left|u_{-}\right|,\left|u_{+}\right|\right\}$and $\bar{v}=\max \left\{\left|v_{-}\right|,\left|v_{+}\right|\right\}$, it follows from $\left|\mu v_{+}\right| \leq \bar{v}$ and $|u(\tau)|,\left|\mu u_{+}\right| \leq \bar{u}$ that the right-hand side of (2.17) is bounded independently of $L \geq 1$ and $\mu \in[0,1]$. Therefore, inequality (2.17) establishes estimate (2.3) if $\tau \geq 1$.

Suppose now that $0 \leq \tau<1$. Then, integrating the second equation of (2.1) over $(\tau, \xi), \xi \in(\tau, 1)$, we obtain

$$
0 \geq \epsilon v^{\prime}(\xi) \geq \mu g(u(\xi))-\mu g(u(\tau))
$$

Moreover, integrating (2.18) over $(\tau, 1)$, we obtain

$$
0 \geq \epsilon(v(1)-v(\tau)) \geq \mu \int_{\tau}^{1} g(u(\xi)) d \xi-\mu g(u(\tau))(1-\tau)
$$

Inequality (2.19) shows that $v(1)-v(\tau)$ is bounded independently of $L \geq 1$ and $\mu \in[0,1]$, so that, with the help of (2.17), we have estimate (2.3) if $0 \leq \tau<1$. Thus estimate (2.3) in Case 1 is proved.

Next, we prove estimate (2.3) in Case 2. We only prove the case that $v(\xi)$ is strictly increasing on $(-L, L)$, because the case that $v(\xi)$ is strictly decreasing on $(-L, L)$ is proved by arguments similar to the proof of the case that $v(\xi)$ is strictly increasing on $(-L, L)$. Note that when $v(\xi)$ is strictly increasing on $(-L, L), u(\xi)$ is strictly increasing on $(-L, \tau)$, attains a maximum at $\tau$, and is strictly decreasing on $(\tau, L)$. Thus, in this case, it is sufficient to estimate $u(\tau)$ from above. In estimating $u(\tau)$ from above, we assume that $u(\tau)>\bar{u} \geq 0$, because it is clear that estimate (2.3) in the case of $u(\tau) \leq \bar{u}$ holds.

We fix $\xi_{0}<\tau$ such that $u\left(\xi_{0}\right) \geq \bar{u}$, and let $\bar{\xi}$ denote the point in $(\tau, L]$ with the property $u(\bar{\xi})=u(\xi)$ for any $\xi \in\left[\xi_{0}, \tau\right)$. Integrating the first equation in (2.1) over $(\xi, \bar{\xi})$, we obtain

$$
\epsilon u^{\prime}(\bar{\xi})-\epsilon u^{\prime}(\xi)=-\mu v(\bar{\xi})+\mu v(\xi)-\int_{\xi}^{\bar{\xi}} \zeta u^{\prime}(\zeta) d \zeta .
$$

Noting that $\xi \in\left[\xi_{0}, \tau\right)$, since $u^{\prime}(\bar{\xi}) \leq 0$ and

$$
-\int_{\xi}^{\bar{\xi}} \zeta u^{\prime}(\zeta) d \zeta=\int_{\xi}^{\bar{\xi}}(u(\zeta)-u(\xi)) d \zeta \geq 0
$$


inequality (2.20) gives

$$
\epsilon u^{\prime}(\xi) \leq \mu v(\bar{\xi})-\mu v(\xi) \leq 2 \bar{v} \leq 2 \bar{v}(1+u(\xi))^{p} \quad \text { for } 1<p<2 .
$$

Integrating (2.21) over $\left(\xi_{0}, \tau\right)$, we obtain

$$
(u(\tau)+1)^{1-p} \geq\left(u\left(\xi_{0}\right)+1\right)^{1-p}-2(p-1) \bar{v} \epsilon^{-1}\left(\tau-\xi_{0}\right) .
$$

Our goal is to show that, for an appropriate choice of $\xi_{0}$, the right-hand side of (2.22) is bounded from below by a positive constant $\Lambda$ which depends solely on $\epsilon, f, g, \bar{u}, \bar{v}$ (and thus, is independent of $L \geq 1$ and $\mu \in[0,1])$. Indeed, from this fact we can easily check that

$$
u(\tau) \leq \Lambda^{\frac{1}{1-p}}-1,
$$

which means that estimate (2.3) holds.

Let $\xi^{*}<\tau$ be the point with $u\left(\xi^{*}\right)=\bar{u}$. Then $\xi_{0}$ must lie in the interval $\left[\xi^{*}, \tau\right)$. To prove that the right-hand side of (2.22) is bounded from below by a positive constant $\Lambda$, we now distinguish between the case satisfying

$$
2(p-1) \bar{v} \epsilon^{-1}\left(\tau-\xi^{*}\right)<\frac{1}{2}(\bar{u}+1)^{1-p},
$$

and the case satisfying

$$
2(p-1) \bar{v} \epsilon^{-1}\left(\tau-\xi^{*}\right) \geq \frac{1}{2}(\bar{u}+1)^{1-p} .
$$

If inequality (2.24) holds, then we select $\xi_{0}=\xi^{*}$ and observe that the right-hand side of (2.22) is bounded from below by

$$
\Lambda=\frac{1}{2}(\bar{u}+1)^{1-p}>0,
$$

while if inequality (2.25) holds, then we select

$$
\xi_{0}=\tau-\min \left\{\frac{\epsilon(\bar{u}+1)^{1-p}}{4(p-1) \bar{v}}, \frac{2 \bar{v}}{\bar{u}+1},\left[\frac{\epsilon(4 \bar{v})^{1-p}}{4(p-1) \bar{v}}\right]^{\frac{1}{2-p}}\right\} .
$$

By the geometry of $v(\xi)$, it is noticed that $\bar{v}>0$. With this choice of $\xi_{0}$, using (2.8) and recalling that $1<p<2$, one easily deduces that the right-hand side of (2.22) is bounded from below by

$$
\begin{aligned}
\Lambda & =\frac{1}{2}(4 \bar{v})^{1-p}\left(\tau-\xi_{0}\right)^{p-1} \\
& \geq \frac{1}{2}(4 \bar{v})^{1-p}\left(\min \left\{\frac{\epsilon(\bar{u}+1)^{1-p}}{4(p-1) \bar{v}}, \frac{2 \bar{v}}{\bar{u}+1},\left[\frac{\epsilon(4 \bar{v})^{1-p}}{4(p-1) \bar{v}}\right]^{\frac{1}{2-p}}\right\}\right)^{p-1}>0 .
\end{aligned}
$$

Thus estimate (2.3) in Case 2 is proved, and the proof of Theorem 2.1 is complete. 
3. Existence of solutions to the Riemann problem. In this section, we prove the main result on the existence of solutions to the Riemann problem for system (1.1) satisfying (1.13), (1.14) and (1.15).

Theorem 3.1. Assume that conditions (1.13), (1.14) and (1.15) are satisfied. Then there exists a solution to the Riemann problem for system (1.1).

This theorem follows from the following theorem, which is established in Theorem 3.2 of Dafermos 2], under the assumption that the solution has a uniformly bounded variation:

Theorem 3.2. For every $\epsilon>0$, let $\left(u_{\epsilon}(\xi), v_{\epsilon}(\xi)\right)$ denote a solution to (1.18), (1.19). Suppose that the set $\left\{\left(u_{\epsilon}(\cdot), v_{\epsilon}(\cdot)\right): 0<\epsilon \leq 1\right\}$ is of uniformly bounded variation. Then there exists a solution to the Riemann problem for system (1.1).

By applying Theorem 3.2, we can prove Theorem 3.1, which means the existence of solutions to the Riemann problem for system (1.1). From Theorems 2.3 and 3.2, it is sufficient for the proof of Theorem 3.1 to prove

$$
\left|u_{\epsilon}(\xi)\right|, \quad\left|v_{\epsilon}(\xi)\right| \leq M \quad \text { for all } \xi \in(-\infty, \infty),
$$

for a nonnegative constant $M$ which is independent of $\epsilon$. By Theorem 2.3, at least one of $u_{\epsilon}(\xi), v_{\epsilon}(\xi)$ is monotone. If both $u_{\epsilon}(\xi)$ and $v_{\epsilon}(\xi)$ are monotone, then inequality (3.1) clearly holds. Therefore, it is sufficient for the proof of inequality (3.1) to deal with the following two cases:

CASE 1. $u_{\epsilon}(\xi)$ is strictly increasing (or decreasing) on $(-\infty, \infty)$, while $v_{\epsilon}(\xi)$ is strictly increasing (or decreasing) on $\left(-\infty, \tau_{\epsilon}\right)$, attains a maximum (or minimum) at $\tau_{\epsilon}$, and is strictly decreasing (or increasing) on $\left(\tau_{\epsilon}, \infty\right)$.

CASE 2. $u_{\epsilon}(\xi)$ is strictly increasing (or decreasing) on $\left(-\infty, \tau_{\epsilon}\right)$, attains a maximum (or minimum) at $\tau_{\epsilon}$, and is strictly decreasing (or increasing) on $\left(\tau_{\epsilon}, \infty\right)$, while $v_{\epsilon}(\xi)$ is strictly increasing (or decreasing) on $(-\infty, \infty)$.

We now prove Theorem 3.1 by proving inequalities (3.1) in Cases 1 and 2 .

First, we prove inequality (3.1) in Case 1 . We only prove the case that $u_{\epsilon}(\xi)$ is strictly increasing on $(-\infty, \infty)$, because the case that $u_{\epsilon}(\xi)$ is strictly decreasing on $(-\infty, \infty)$ is proved by arguments similar to the proof of the case that $u_{\epsilon}(\xi)$ is strictly increasing on $(-\infty, \infty)$. Note that when $u_{\epsilon}(\xi)$ is strictly increasing on $(-\infty, \infty), v_{\epsilon}(\xi)$ is strictly increasing on $\left(-\infty, \tau_{\epsilon}\right)$, attains a maximum at $\tau_{\epsilon}$, and is strictly decreasing on $\left(\tau_{\epsilon}, \infty\right)$. Thus, in this case, it is sufficient to estimate $v_{\epsilon}\left(\tau_{\epsilon}\right)$ from above.

Suppose first that $\left|\tau_{\epsilon}\right| \leq 1$. Note that for every $\epsilon>0$, there exists $\xi_{\epsilon} \in\left(\tau_{\epsilon}+1, \tau_{\epsilon}+2\right)$ such that

$$
u_{\epsilon}^{\prime}\left(\xi_{\epsilon}\right) \leq u_{+}-u_{-} .
$$

Then, integrating the first equation in (1.18) over $\left(\tau_{\epsilon}, \xi_{\epsilon}\right)$, and using $u_{\epsilon}^{\prime}\left(\tau_{\epsilon}\right) \geq 0$ and (3.2), we obtain

$$
v_{\epsilon}\left(\tau_{\epsilon}\right) \leq f\left(u_{\epsilon}\left(\tau_{\epsilon}\right)\right)-f\left(u_{\epsilon}\left(\xi_{\epsilon}\right)\right)+v_{\epsilon}\left(\xi_{\epsilon}\right)+(\epsilon+3)\left(u_{+}-u_{-}\right) .
$$

On account of (2.5), the right-hand side of (3.3) is bounded independently of $0<\epsilon \leq 1$. Thus if $\left|\tau_{\epsilon}\right| \leq 1$, then inequality (3.1) in Case 1 holds. 
Next, suppose that $\left|\tau_{\epsilon}\right|>1$. Since the proof of the case of $\tau_{\epsilon}<-1$ is similar to the proof of the case of $\tau_{\epsilon}>1$, we may suppose that $\tau_{\epsilon}>1$. Then, for any $k>\tau_{\epsilon}$, integrating the second equation in (1.18) over $\left(\tau_{\epsilon}, k\right)$, we have

$$
\epsilon v_{\epsilon}{ }^{\prime}(k)-\epsilon v_{\epsilon}{ }^{\prime}\left(\tau_{\epsilon}\right)=g\left(u_{\epsilon}(k)\right)-g\left(u_{\epsilon}\left(\tau_{\epsilon}\right)\right)+\tau_{\epsilon}\left(v_{\epsilon}\left(\tau_{\epsilon}\right)-v_{\epsilon}(k)\right)+\int_{\tau_{\epsilon}}^{k} v_{\epsilon}(\xi)-v_{\epsilon}(k) d \xi
$$

so that

$$
0>g\left(u_{\epsilon}(k)\right)-g\left(u_{\epsilon}\left(\tau_{\epsilon}\right)\right)+\tau_{\epsilon}\left(v_{\epsilon}\left(\tau_{\epsilon}\right)-v_{\epsilon}(k)\right) .
$$

Passing to $k \rightarrow \infty$ in (3.4), we obtain

$$
0 \geq g\left(u_{+}\right)-g\left(u_{\epsilon}\left(\tau_{\epsilon}\right)\right)+\tau_{\epsilon}\left(v_{\epsilon}\left(\tau_{\epsilon}\right)-v_{+}\right) .
$$

Therefore, we have

$$
v_{\epsilon}\left(\tau_{\epsilon}\right) \leq \frac{g\left(u_{\epsilon}\left(\tau_{\epsilon}\right)\right)-g\left(u_{+}\right)}{\tau_{\epsilon}}+v_{+} \leq g\left(u_{\epsilon}\left(\tau_{\epsilon}\right)\right)-g\left(u_{+}\right)+v_{+} .
$$

Thus inequality (3.1) in Case 1 is proved.

We proceed to prove inequality (3.1) in Case 2. We only prove the case that $v_{\epsilon}(\xi)$ is strictly increasing on $(-\infty, \infty)$, because the case that $v_{\epsilon}(\xi)$ is strictly decreasing on $(-\infty, \infty)$ is proved by arguments similar to the proof of the case that $v_{\epsilon}(\xi)$ is strictly increasing on $(-\infty, \infty)$. Note that when $v_{\epsilon}(\xi)$ is strictly increasing on $(-\infty, \infty), u_{\epsilon}(\xi)$ is strictly increasing on $\left(-\infty, \tau_{\epsilon}\right)$, attains a maximum at $\tau_{\epsilon}$, and is strictly decreasing on $\left(\tau_{\epsilon}, \infty\right)$. Thus, in this case, it is sufficient to estimate $u_{\epsilon}\left(\tau_{\epsilon}\right)$ from above.

Note that for every $\epsilon>0$, there exist $\zeta_{\epsilon} \in\left(\tau_{\epsilon}-2, \tau_{\epsilon}-1\right)$ and $\xi_{\epsilon} \in\left(\tau_{\epsilon}+1, \tau_{\epsilon}+2\right)$ such that

$$
v_{\epsilon}^{\prime}\left(\zeta_{\epsilon}\right) \leq v_{+}-v_{-}, \quad v_{\epsilon}^{\prime}\left(\xi_{\epsilon}\right) \leq v_{+}-v_{-} .
$$

Then, integrating the second equation in (1.18) over $\left(\tau_{\epsilon}, \xi_{\epsilon}\right)$, and using $v_{\epsilon}^{\prime}\left(\tau_{\epsilon}\right) \geq 0$ and (3.7), we obtain

$$
g\left(u_{\epsilon}\left(\tau_{\epsilon}\right)\right) \geq g\left(u_{\epsilon}\left(\xi_{\epsilon}\right)\right)-(\epsilon+2)\left(v_{+}-v_{-}\right)-\left|\tau_{\epsilon}\right|\left(v_{\epsilon}\left(\xi_{\epsilon}\right)-v_{\epsilon}\left(\tau_{\epsilon}\right)\right) .
$$

Moreover, integrating the second equation in (1.18) over $\left(\zeta_{\epsilon}, \xi_{\epsilon}\right)$, we have

$$
\begin{aligned}
\tau_{\epsilon}\left(v_{\epsilon}\left(\zeta_{\epsilon}\right)-v_{\epsilon}\left(\xi_{\epsilon}\right)\right)=\epsilon v^{\prime}\left(\xi_{\epsilon}\right) & -\epsilon v^{\prime}\left(\zeta_{\epsilon}\right)-g\left(u_{\epsilon}\left(\xi_{\epsilon}\right)\right)+g\left(u_{\epsilon}\left(\zeta_{\epsilon}\right)\right) \\
& +\left(\tau_{\epsilon}-\zeta_{\epsilon}\right)\left(v_{\epsilon}\left(\zeta_{\epsilon}\right)-v_{\epsilon}\left(\xi_{\epsilon}\right)\right)-\int_{\zeta_{\epsilon}}^{\xi_{\epsilon}} v_{\epsilon}(\xi)-v_{\epsilon}\left(\xi_{\epsilon}\right) d \xi
\end{aligned}
$$

so that

$$
\left|\tau_{\epsilon}\right|\left(v_{\epsilon}\left(\xi_{\epsilon}\right)-v_{\epsilon}\left(\tau_{\epsilon}\right)\right) \leq(6+2 \epsilon)\left(v_{+}-v_{-}\right)+\left|g\left(u_{\epsilon}\left(\xi_{\epsilon}\right)\right)\right|+\left|g\left(u_{\epsilon}\left(\zeta_{\epsilon}\right)\right)\right| .
$$

On account of (2.8), the right-hand sides of (3.8) and (3.10) are bounded independently of $0<\epsilon \leq 1$. Therefore, by conditions (1.14) and (1.15), we see that $u_{\epsilon}\left(\tau_{\epsilon}\right)$ is bounded, independently of $0<\epsilon \leq 1$, from above. Thus inequality (3.1) in Case 2 is proved and the main result of this paper, Theorem 3.1, is now fully proved. 


\section{REFERENCES}

[1] A. Bressan, Hyperbolic Systems of Conservation Laws, Oxford University Press, Oxford, 2000. MR $1816648(2002 \mathrm{~d}: 35002)$

[2] C. M. Dafermos, Solution of the Riemann problem for a class of hyperbolic conservation laws by the viscosity method, Arch. Rational Mech. Anal. 52 (1973), 1-9. MR0340837 (49:5587)

[3] C. M. Dafermos, The entropy rate admissibility criterion for solutions of hyperbolic conservation laws, J. Differential Equations 14 (1973), 202-212. MR0328368 (48:6710)

[4] C. M. Dafermos, Structure of solutions of the Riemann problem for hyperbolic systems of conservation laws, Arch. Rational Mech. Anal. 53 (1974), 203-217. MR0348289 (50:787)

[5] C. M. Dafermos and R. J. DiPerna, The Riemann problem for certain classes of hyperbolic systems of conservation laws, J. Differential Equations 20 (1976), 90-114. MR0404871 (53:8671)

[6] H.-T. Fan, A limiting "viscosity" approach to the Riemann problem for materials exhibiting changes of phase (II), Arch. Rational Mech. Anal. 116 (1992), 317-337. MR.1132765 (93a:35102)

[7] H.-T. Fan, One-phase Riemann problem and wave interactions in systems of conservation laws of mixed type, SIAM J. Math. Anal. 24 (1993), 840-865. MR.1226854 (94f:35082)

[8] H.-T. Fan, A vanishing viscosity approach on the dynamics of phase transitions in van der Waals fluids, J. Differential Equations 103 (1993), 179-204. MR.1218743 (94g:35140)

[9] H.-T. Fan and M. Slemrod, Dynamic flows with liquid/vapor phase transitions, Handbook of mathematical fluid dynamics, Vol. I, 373-420, North-Holland, Amsterdam, 2002. MR1942467 (2003j:76085)

[10] B. L. Keyfitz and H. C. Kranzer, Existence and uniqueness of entropy solutions to the Riemann problem for hyperbolic systems of two nonlinear conservation laws, J. Differential Equations 27 (1978), 444-476. MR0466993(57:6866)

[11] B. L. Keyfitz and H. C. Kranzer, A system of nonstrictly hyperbolic conservation laws arising in elasticity theory, Arch. Rational Mech. Anal. 72 (1980), 219-241. MR549642 (80k:35050)

[12] B. L. Keyfitz and H. C. Kranzer, The Riemann problem for a class of hyperbolic conservation laws exhibiting a parabolic degeneracy, J. Differential Equations 47 (1983), 35-65. MR684449)(84a:35162)

[13] P. D. Lax, Hyperbolic systems of conservation laws II, Comm. Pure Appl. Math. 10 (1957), 537-566. MR0093653 (20:176)

[14] T. P. Liu, The Riemann problem for general $2 \times 2$ conservation laws, Trans. Amer. Math. Soc. 199 (1974), 89-112. MR0367472 (51:3714)

[15] T. P. Liu, Existence and uniqueness theorems for Riemann problems, Trans. Amer. Math. Soc. 212 (1975), 375-382. MR0380135(52:1036)

[16] H. Ohwa, On shock curves in $2 \times 2$ hyperbolic systems of conservation laws, Adv. Math. Sci. Appl. 19 (2009), 227-244. MR2553478 (2010i:35236)

[17] H. Ohwa, The shock curve approach to the Riemann problem for $2 \times 2$ hyperbolic systems of conservation laws, J. Hyperbolic Differ. Equ. 7 (2010), 339-364. MR.2659740

[18] H. Ohwa, On the uniqueness of entropy solutions to the Riemann problem for $2 \times 2$ hyperbolic systems of conservation laws, Commun. Math. Sci. 9 (2011), 161-185.

[19] M. Slemrod, A limiting "viscosity" approach to the Riemann problem for materials exhibiting change of phase, Arch. Rational Mech. Anal. 105 (1989), 327-365. MR.973246 (89m:35186)

[20] J. A. Smoller, On the solution of the Riemann problem with general step data for an extended class of hyperbolic systems, Michigan Math. J. 16 (1969), 201-210. MR0247283 (40:552)

[21] V. A. Tupciev, On the method of introducing viscosity in the study of problems involving the decay of discontinuity, Dokl. Akad. Nauk. SSSR 211 (1973), 55-58. MR0330801 (48:9138) 